\title{
A Case Report of Abdominal Pregnancy
}

\author{
Dr. B. Anil Kumar ${ }^{1}$, Dr. K. Jaya Prada Devi², Dr. M. Aparna ${ }^{3}$, \\ Dr. V. Krishna Chaitanya ${ }^{4}$, \\ ${ }^{1,3,4}$ (Dept. of General Surgery, Dr. PSIMS \& RF, Chinoutpalli, AP, India) \\ ${ }^{2}$ (Dept. of OBG, Dr. PSIMS \& RF, Chinoutpalli, AP, India)
}

\begin{abstract}
Abdominal pregnancy is an extremely rare form of extra uterine gestation. They may be primary or secondary with USG and MRI aiding in the diagnosis. Here we report a case of abdominal pregnancy in a 24 year woman with 12 wks amenorrhoea.
\end{abstract}

\section{Introduction}

Ectopic and extra uterine pregnancy refers to implantation of blastocyst or fertilized ovum outside the uterine cavity. The commonest sites of such pregnancies are fallopian tubes and the least frequent being the ovary and abdominal cavity.

Abdominal pregnancy, with a diagnosis of one per 10000 births, is an extremely rare and serious form of extrauterine gestation ${ }^{[1]}$. Abdominal pregnancies account for almost $1 \%$ of ectopic pregnancies ${ }^{[2]}$. The incidence of abdominal pregnancy varies from 1 in 372 to 1 in 9,714 live births. Abdominal pregnancy is associated with high morbidity and mortality, with the risk for death 7 to 8 times greater than from tubal ectopic pregnancy and 50 times greater than from intrauterine pregnancy ${ }^{[3]}$. The high rate of morbidity and mortality from abdominal pregnancy often results from a delay in diagnosis. The placenta can be attached to the uterine wall, bowel, mesentery, liver, spleen, bladder and ligaments. It can be detach at any time during pregnancy leading to torrential blood loss ${ }^{[4]}$.

Abdominal pregnancies are classified as primary or secondary. Most are secondary, the result of early tubal abortion or rupture with secondary implantation of the pregnancy into the peritoneal cavity. Sonography is considered the front-line diagnostic imaging method, with magnetic resonance imaging (MRI) serving as an adjunct in cases when sonography is equivocal and in cases when the delineation of anatomic relationships may alter the surgical approach ${ }^{[5]}$. Here we report a case of abdominal pregnancy.

\section{Case report}

A 24 year old Mrs. $\mathrm{x}$ was admitted in the emergency department of Dr. PSIMS \& RF with c/o diffuse pain abdomen of 2 days duration. She had a history of $12 \mathrm{Wks}$ amenorrhoea followed by 2 episodes of bleeding $\mathrm{p} / \mathrm{v} 10$ days apart. She gave a H/o dilatation \& curretage 10 days back after the second bleeding episode.

No USG was done at that time. She had a full term vaginal delivery 10 months back with outcome of a healthy live male child. There was no H/o contraceptive usage. She had a H/o appendectomy 2 years back.

On examination, she was found to have severe pallor with tachycardia. Her blood pressure was $80 / 50 \mathrm{mmHg}$. There was marked tenderness along with guarding and rigidity of the lower abdomen. Pelvic examination revealed normal size uterus with marked tenderness in left fornix and no other palpable masses. Urine pregnancy test positive. Ultrasound examination revealed bulky uterus with empty cavity with fetus of 10 weeks seen in the abdominal cavity. Free fluid was also present in the abdominal cavity.5

Emergency laparotomy was done. Intra operative findings: ectopic pregnancy in the left broad ligament forming a cystic mass was seen. Hemoperitoneum of 2 liters was present. The uterus was slightly enlarged, right fallopian tube and ovary appeared normal. Left tube and ovary appeared normal but left fallopian tube and ovary were adherent to the mass closely. Left salpingectomy with surgical removal of ectopic pregnancy was done. The patient was transfused three units of blood. Patient had an uneventful recovery and was discharged on the sixth postoperative day. 


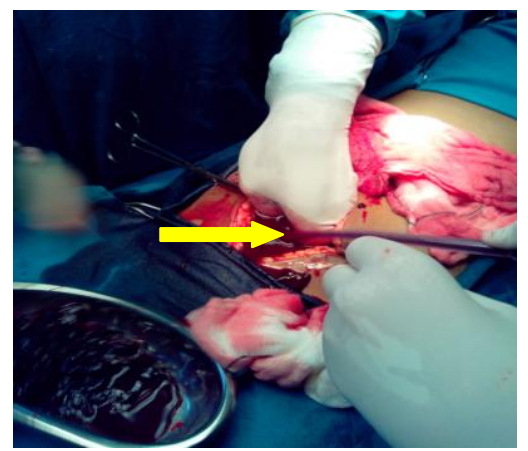

Fig 1: Haemoperitoneum

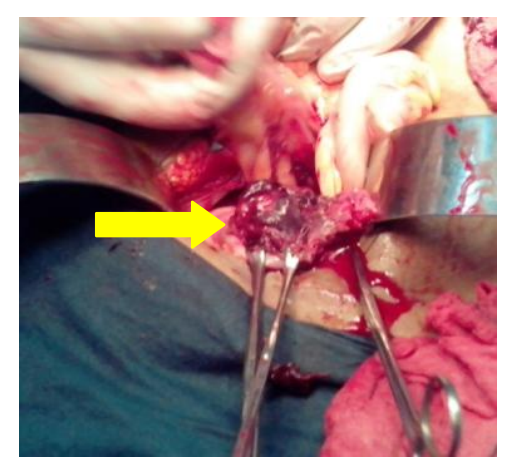

Fig 2: Ectopic pregnancy in broad ligament.

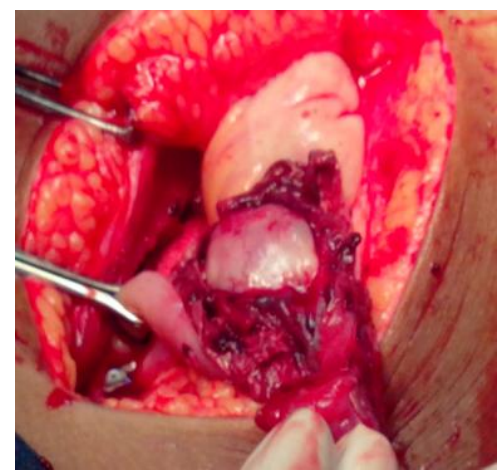

Fig 3

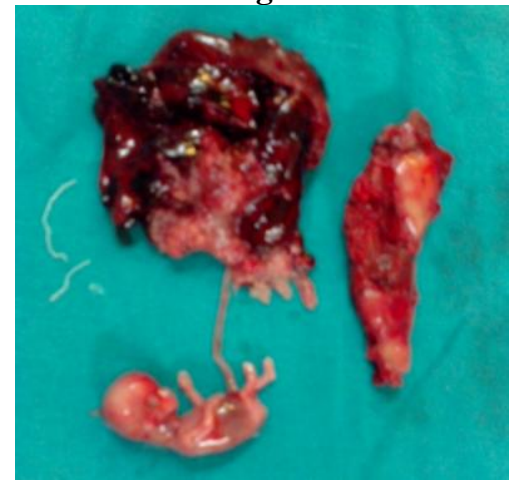

Fig 4

\section{Discussion} mortality.

Abdominal pregnancy is a rare form of ectopic pregnancy with increased maternal morbidity and

Documented risk factors such as previous ectopic pregnancy, Tubal corrective surgery, Tubal sterilization, Intrauterine device usage, documented tubal pathology, Infertility Assisted reproductive 
technology, Previous genital infection, Smoking, Prior abortion, Multiple sexual partners and Prior cesarean delivery were not present in this case. The presentation of patients with an abdominal pregnancy varies and depends on the gestational age. In the first and early second trimester, the symptoms may be the same as with tubal ectopic gestation; in advanced abdominal pregnancy, the clinical presentation is more variable. The patient may report painful fetal movement, fetal movements high in the abdomen, or sudden cessation of movements. Physical examination may disclose persistent abnormal fetal positioning, abdominal tenderness, a displaced uterine cervix, easy palpation of fetal parts, and palpation of the uterus separate from the gestation. Our patient presented with bleeding $\mathrm{p} / \mathrm{v}$ and pain abdomen.

In a study of 12 patients reported by Hallatt and Grove ${ }^{[7]}$, vaginal bleeding occurred in six patients. Ultrasound examination is the usual diagnostic procedure of choice, but the findings are sometimes questionable. Transvaginal ultrasound is superior to transabdominal ultrasound. MRI provided additional information for patients who needed precise diagnosing. After the diagnosis of abdominal pregnancy became definitive, it was essential to determine the localization of the placenta. Meanwhile, MRI may help in surgical planning by evaluating the extent of mesenteric and uterine involvement ${ }^{[7]}$. Removal of the placental tissue is less difficult in early pregnancy as it is likely to be smaller and less vascular. Complete removal of the placenta should be done only when the blood supply can be identified and careful ligation performed ${ }^{[6]}$. If the placenta is not removed completely, it has been estimated that the remnant can remain functional for approximately 50 days after the operation, and total regression of placental function is usually complete within 4 months. ultrasound scanning plus MRI can be useful to demonstrate the anatomic relationship between the placenta and invasion area in order to be prepared preoperatively for the possible massive blood loss. Our case was diagnosed on USG and managed by exploratory laparotomy.

\section{Conclusion}

Ectopic pregnancy in any form is associated with high mortality and morbidity. High index of suspicion is necessary in reproductive age group women presenting with pain abdomen and bleeding $p / v$ following a period of amenorrhoea. Prompt diagnosis with effective management reduces morbidity and mortality. Ultrasound is mandatory in a patient with amenorrhoea prior to D \& C when urine pregnancy test is positive.

\section{References}

[1]. Yildizhan R, Kurdoglu M, Kolusari A, Erten R: Primary omental pregnancy. Saudi Med J 2008, 29:606-609.

[2]. Mc Graw Hill Williams OBSTETRICS $23^{\text {rd }}$ ed ch $10 ;$ p237

[3]. Amy J. Voedisch, Carrie E. Frederick, Antonia F. Nicosia, Thomas G. Stovall: Early pregnancy loss and Ectopic pregnancy in wolters kluwer/lippincott williams \& wilkins, Berek \& Novak's Gynaecology $15^{\text {th }}$ ed ; p644

[4]. Ang LP, Tan AC, Yeo SH: Abdominal pregnancy: a case report and literature review. Singapore Med J 2000, 41:454-457.

[5]. Wagner A, Burchardt A: MR imaging in advanced abdominal pregnancy. Acta Radiol 1995, 36:193-195

[6]. Hallatt JG, Grove JA: Abdominal pregnancy: a study of twenty one consecutive cases. Am J Obstet Gynecol 1985, 152:444-449.

[7]. Malian V, Lee JH: MR imaging and MR angiography of an abdominal pregnancy with placental infarction. AJR Am JRoentgenol 2001, 177:1305-1306. 